\title{
Examining the Effects of Lighting Effects on Peripheral Devices for Visual User Notifications
}

\author{
Brandon JC Scott \\ Computing and Informatics \\ Bournemouth University, UK \\ bscott@bournemouth.ac.uk
}

\author{
Huseyin Dogan \\ Computing and Informatics \\ Bournemouth University, UK \\ hdogan@bournemouth.ac.uk
}

\author{
Nan Jiang \\ Computing and Informatics \\ Bournemouth University, UK \\ njiang@bournemouth.ac.uk
}

\begin{abstract}
The ubiquitous and pervasive use of lighting effects embedded into peripheral hardware has gained popularity through it's use in Triple-A video game titles such as Call of Duty and the availability of software development kits (SDK) from leading manufacturers. A preliminary NASA TLX experiment was performed to examine the effect that notifications displayed on a peripheral device has, in comparison to traditional dialog notifications. This research will prove useful to create notification design guidance for these devices.
\end{abstract}

Notifications, Peripherals, HCl, UX, Human Factors, NASA TLX, Video Games

\section{INTRODUCTION}

Notifications in all contexts are inherently distracting. The purpose of a notification is to alert a user to current information or a predefined event. A user must register or acknowledge these prompts for them to be effective (McCrickard et al. 2003). If a user decides to change task based on a notification, it can be detrimental to the original focus and increase time required for the original task output (Kasai et al. 2005). The user-experience of this scenario unfavourable, demonstrating a lack of standard notification design practice across software platforms.

Peripheral hardware with back-lighting is common nowadays, with a primary use case of allowing users to work in a dark environment. The popularity of such devices grew when it became apparent that video game players, preferred to play in low lighting conditions, as it allows for a more immersive experience (Nordin et al. 2009) With recent advances in the field of peripheral devices, the integration of lighting effects has made it possible to provide visual indication output, a medium that has previously provided only user input capabilities. There are a variety of different use cases including the ability for software to interact with users through notifications on keyboards, mice and gamepads. Whereas existing applications alert users using traditional on-screen dialogs, visual user notifications (through lighting effects)

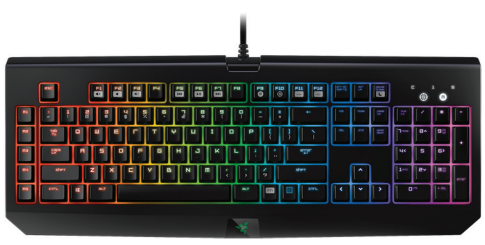

Figure 1: A keyboard with individual backlit LEDs under each key

on peripheral devices may provide a less intrusive experience reducing cognitive load. Additionally, the customisability of these lighting effects can be considered an extension of a game or application to further enhance gaming experiences. The following research question is put forward: Are peripheral lighting based notifications less distracting than traditional dialog based notifications and to what extent do users feel distracted when using notifications?

\section{METHOD}

Otherwise known as an auxiliary device, a peripheral is a physical appliance that provides input/output (I/O) from a computer (Laplante 2000). Some manufacturers have now developed hardware that provide lighting capabilities on these types of devices (see figure 1). This provides an output medium which is separate from a screen yet still within the user's peripheral vision. 
As a subjective workload assessment tool, NASA Task Load Index (abbreviated NASA TLX) provides useful insight into a user's cognitive process whilst undertaking a task. This method was selected due to it's multi-dimensional scale, efficiency of data gathering and popularity in industry (Hart 2006). Five participants were selected for this preliminary study (with results shown in Table 1 and 2 ) in an age range of 16-22. A comparative assessment experiment was developed to examine the effect of dialog prompt notifications in comparison to lighting effects present on peripheral devices. For the purpose of this study, an application was written to fire two different notification styles. The first style opened a dialog box on screen whilst the second style flickered the colour of the F1 key on the keyboard between blue and red. Each notification style was timed to execute every 25 seconds. Each participant was asked to run two typing tests, with a duration of two minutes each. After each test, the participant was asked to immediately fill in a paper version of the NASA TLX assessment. Some studies have shown this to reduce cognitive overhead on the users in comparison to a software-based recording system (Cao et al. 2009).

Pilot testing was conducted prior to the main test run to ensure that any problems were identified (Schade 2015). The duration of the test was too long and concentration diminished and thus the timing was reduced. Additionally, the participant was originally asked to use the same text for both tests, however they commented that this was tedious and they would feel more engaged if they could select their own text from the list. As such, participants in the preliminary study were able to select their own texts. The following aspects are measured in NASA TLX with a value between 1 - 21: Mental Demand [MD]; Physical Demand [PD]; Temporal Demand [TD]; Performance [P]; Effort [E]; Frustration [F].

\section{RESULTS AND DISCUSSION}

The results from the comparative workload assessment show differences between the each test undertaken. The main change is between the two frustration results, with a minimum variation of 10 gradations and a maximum of 14 gradations.

As shown in figure 2, there is a correlation between the two tests each user undertook. This demonstrates that users who feel more frustrated in test one, feel less frustrated by test two. There is also correlation shown between temporal demand and frustration. This is exemplified by Participant 5 where for Test 1 , they rated their temporal demand as $\mathbf{2 1}$ and frustration 17. This is a stark difference from Test 2 , where the same participant rated their temporal

\begin{tabular}{lcccccc}
\hline & MD & PD & TD & P & E & F \\
\hline Participant 1 & 3 & 1 & 6 & 14 & 21 & 17 \\
Participant 2 & 4 & 1 & 6 & 7 & 21 & 15 \\
Participant 3 & 7 & 3 & 18 & 7 & 14 & 17 \\
Participant 4 & 14 & 10 & 21 & 12 & 14 & 21 \\
Participant 5 & 4 & 1 & 21 & 18 & 5 & 17 \\
\hline
\end{tabular}

Table 1: Dialog Based Notification Results

\begin{tabular}{ccccccc}
\hline & MD & PD & TD & P & E & F \\
\hline Participant 1 & 3 & 1 & 6 & 13 & 21 & 6 \\
Participant 2 & 4 & 1 & 11 & 6 & 21 & 5 \\
Participant 3 & 7 & 4 & 12 & 10 & 7 & 5 \\
Participant 4 & 10 & 6 & 21 & 17 & 10 & 7 \\
Participant 5 & 10 & 1 & 4 & 1 & 4 & 4 \\
\hline
\end{tabular}

Table 2: Peripheral Based Notification Results

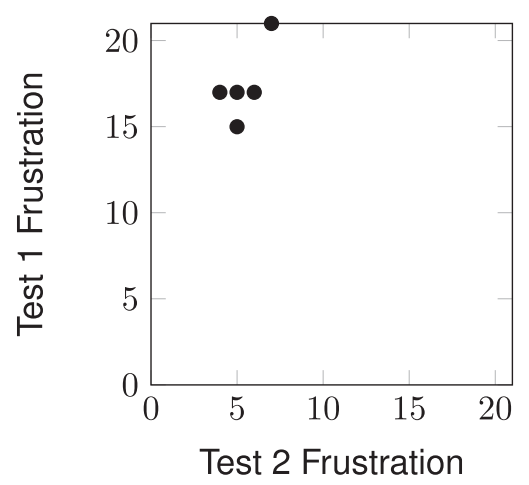

Figure 2: Comparison of participant NASA TLX frustration scores between Test 1 and Test 2

demand as $\mathbf{4}$ and frustration as $\mathbf{4}$. It could therefore be said, that users find dialog-based notifications not only more frustrating, but that they feel more rushed or hurried due to it.

\section{CONCLUSION AND FUTURE WORK}

It can be said that there is a marked difference in the frustration levels between the two tasks carried out in the comparative workload assessment by a minimum of ten gradations on the NASA TLX scale. Peripheral lighting effects proved to be a less frustrating alert than traditional dialog notifications as exemplified in figure 2. Future work could be carried out to quantify this data (given that NASA TLX is subjective) using eye-tracking and user logging with a larger set of users. Additionally, research into how frustration affects distraction would prove useful when identifying the temporal demand of a notification on end users. This work would help to create supported design guidance for notifications on these systems. 


\section{REFERENCES}

Cao, a., K. K. Chintamani, a. K. Pandya, and R. D. Ellis (2009). NASA TLX: software for assessing subjective mental workload. Behav Res Methods 41(1), 113-117.

Hart, S. G. (2006). NASA-TASK LOAD INDEX (NASA-TLX); 20 YEARS LATER. In HFES.

Kasai, H., K. Yamazaki, and S. Kurakake (2005). Adaptive notification system guaranteeing message reachability. Proceedings - International Conference on Pervasive Services, ICPS '05 2005(0), 208-217.

Laplante, P. a. (2000). Dictionary of Computer Science, Engineering and Technology. CRC Press.
McCrickard, D. S., M. Czerwinski, and L. Bartram (2003). Introduction: Design and evaluation of notification user interfaces. International Journal of Human Computer Studies 58(5), 509-514.

Nordin, A. I., P. Cairns, M. Hudson, A. Alonso, and E. H. Calvillo (2009). The Effect Of Surroundings On Gaming Experience. In Proceedings of the 9th International Conference on the Foundations of Digital Games (FDG 2014). Society for the Advancement of the Science of Digital Games.

Schade, A. (2015). Pilot Testing: Getting It Right (Before) the First Time. 